\title{
Sulfatase modifying factor 1 (SUMF1) is associated with Chronic Obstructive Pulmonary Disease
}

Julie Weidner', Linnea Jarenbäck ${ }^{1}$, Kim de Jong ${ }^{2}$, Judith M. Vonk ${ }^{2}$, Maarten van den Berge ${ }^{3}$, Corry-Anke Brandsma ${ }^{3}$, H. Marike Boezen², Don Sin ${ }^{4}$, Yohan Bossé ${ }^{5}$, David Nickle ${ }^{6}$, Jaro Ankerst ${ }^{1}$, Leif Bjermer ${ }^{1}$, Dirkje S. Postma ${ }^{3}$, Alen Faiz ${ }^{3}$ and Ellen Tufvesson ${ }^{1 *}$

\begin{abstract}
Background: It has been observed that mice lacking the sulfatase modifying factor (Sumf1) developed an emphysema-like phenotype. However, it is unknown if SUMF1 may play a role in Chronic Obstructive Pulmonary Disease (COPD) in humans. The aim was to investigate if the expression and genetic regulation of SUMF1 differs between smokers with and without COPD.

Methods: SUMF1 mRNA was investigated in sputum cells and whole blood from controls and COPD patients (all current or former smokers). Expression quantitative trait loci (eQTL) analysis was used to investigate if single nucleotide polymorphisms (SNPS) in SUMF1 were significantly associated with SUMF1 expression. The association of SUMF1 SNPS with COPD was examined in a population based cohort, Lifelines. SUMF1 mRNA from sputum cells, lung tissue, and lung fibroblasts, as well as lung function parameters, were investigated in relation to genotype.

Results: Certain splice variants of SUMF1 showed a relatively high expression in lung tissue compared to many other tissues. SUMF1 Splice variant 2 and 3 showed lower levels in sputum cells from COPD patients as compared to controls. Twelve SNPs were found significant by eQTL analysis and overlapped with the array used for genotyping of Lifelines. We found alterations in mRNA expression in sputum cells and lung fibroblasts associated with SNP rs11915920 (top hit in eQTL), which validated the results of the lung tissue eQTL analysis. Of the twelve SNPs, two SNPs, rs793391 and rs308739, were found to be associated with COPD in Lifelines. The SNP rs793391 was also confirmed to be associated with lung function changes.

Conclusions: We show that SUMF1 expression is affected in COPD patients compared to controls, and that SNPs in SUMF1 are associated with an increased risk of COPD. Certain COPD-associated SNPs have effects on either SUMF1 gene expression or on lung function. Collectively, this study shows that SUMF1 is associated with an increased risk of developing COPD.
\end{abstract}

Keywords: Chronic obstructive pulmonary disease, Lung fibroblast, Single nucleotide polymorphism, Sputum, Sulfatase modifying factor 1

\footnotetext{
* Correspondence: Ellen.Tufvesson@med.lu.se

${ }^{1}$ Respiratory Medicine and Allergology, Department of Clinical Sciences Lund,

BMC, D12, Lund University, Skåne University Hospital, 22184 Lund, Sweden

Full list of author information is available at the end of the article
} 


\section{Background}

In recent years, Chronic Obstructive Pulmonary Disease (COPD) has risen to the third leading cause of mortality world-wide [1]. The disease is irreversible and characterized by chronic inflammation around the bronchi and bronchioles leading to fibrosis, tissue destruction, and the development of emphysema. Smoking is the main risk factor for developing COPD, although other environmental factors such as air pollution can also trigger the development of the disease.

Several recent studies have sought to uncover genetic causes of COPD in order to better understand the disease and its progression [2-9]. Through genome-wide association studies (GWAS) and whole genome sequencing, several genes and single nucleotide polymorphisms (SNPs) have been identified as being associated with COPD $[2,3]$. To date, the only known single gene mutation related to COPD is in SERPINA1, which leads to alpha1-antitrypsin deficiency [10]. Multiple cohorts have identified other genes as being associated with COPD susceptibility, but their role in the pathology of the disease remains to be identified [2].

In the lung, the extracellular matrix is important for the proper formation and maintenance of the structure of the alveoli, highlighting the importance of proteoglycans in lung development [11]. Sulfatases act on various cellular substrates, including glycosaminoglycans (GAGs) on proteoglycans, and all sulfatases in the cell are regulated by a single protein, sulfatase modifying factor-1 (SUMF1) [12, 13]. SUMF1 modulates a very specific and unique post-translational modification in the active site of sulfatases [14-17]. Mutations in SUMF1 lead to a variety of human diseases, including effects in the lungs, where an overabundance of sulfated GAGs accumulate [18-20]. To date, there have been no reports on measured GAGs in COPD. Recently, it was observed that a Sumf1-/mouse developed an emphysema-like phenotype following an arrest of alveolarization [21, 22]. It is, however, unknown if SUMF1 may be involved in the development of COPD.

The aim of this study was to examine if SUMF1 is associated with COPD. Primarily we aimed to investigate the SUMF1 expression in COPD patients. By using expression quantitative trait loci (eQTL) analysis, we investigated if SNPs in SUMF1 were associated with SUMF1 expression in lung tissue, and investigated SUMF1 mRNA expression in sputum cells and lung fibroblasts. Thereafter, we examined whether there was a genetic association between SUMF1 and COPD amongst smokers in a population based cohort, and subsequently investigated advanced lung physiology from subjects in the context of the different genotypes.

\section{Methods}

A flowchart diagram (Fig. 1) provides an overview of all the analyses performed in this study investigating the associations between SUMF1 and COPD.

\section{Patients in the Lund cohort}

Forty controls and 82 COPD patients, defined according to GOLD criteria (forced expiratory volume in 1 second $\left(\mathrm{FEV}_{1}\right) /$ forced volume capacity $\left.(\mathrm{FVC})<0.7\right)$, were included in the Lund cohort (Table 1). All subjects were current smokers or ex-smokers with $>15$ pack-years, had normal levels of alpha-1 antitrypsin, and had no history of asthma, lung cancer, or any other cardiorespiratory diseases. They did not suffer from any lower respiratory infections within 3 weeks prior to the visit. They were asked to refrain from inhaled bronchodilators for $8 \mathrm{~h}$ for short acting beta agonists and short acting muscarinic antagonists and $48 \mathrm{~h}$ for long acting beta agonists and long acting muscarinic antagonists before the visit. All study participants performed flow-volume spirometry, body plethysmography (MasterScreen Body, Erich Jaeger $\mathrm{GmbH}$ ), and single breath helium dilution carbon monoxide diffusion (MasterScreen Diffusion, Erich Jaeger $\mathrm{GmbH})$ after bronchodilation (400 $\mu \mathrm{g}$ salbutamol, Buventol Easyhaler $\left.{ }^{\circ}\right)$. Lung function measurements were performed according to manufacturer's instructions and European Respiratory Society/American Thorax Society recommendations [23-25]. The reference values used were established by Crapo et al. [26] (spirometry), and from Quanjer et al. [27] (Body plethysmography and carbon monoxide diffusion). All subjects signed written informed consent and the study was approved by the Regional Ethics Review Board in Lund.

\section{Sputum induction and processing}

Sputum was induced by inhalation of $3 \%$ saline for $5 \mathrm{~min}$, and thereafter $4.5 \%$ saline for $2 \times 5 \mathrm{~min}$. After each step, patients were asked to try to expectorate sputum. Samples were picked for plugs which were incubated with 4 volumes of cold $0.1 \%$ dithiothreitol in phosphate buffered saline. After $30 \mathrm{~min}$ incubation in $4{ }^{\circ} \mathrm{C}$, additional 4 volumes of phosphate buffered saline were added, and the sample was filtered $(60 \mu \mathrm{m}$ filters). Cells were pelleted at $1000 \times g$ for 5 minutes $\left(4{ }^{\circ} \mathrm{C}\right)$ and lysed for future RNA analysis [28].

\section{Lung fibroblasts from biopsies}

A bronchoscopy was performed in 15 COPD patients. Central lung biopsies were sampled from which fibroblasts were isolated as previously published [29].

\section{RNA extraction and qPCR analysis}

For examination of RNA from various body tissues, the Human Total RNA Master Panel II Lot\# 1505145A 


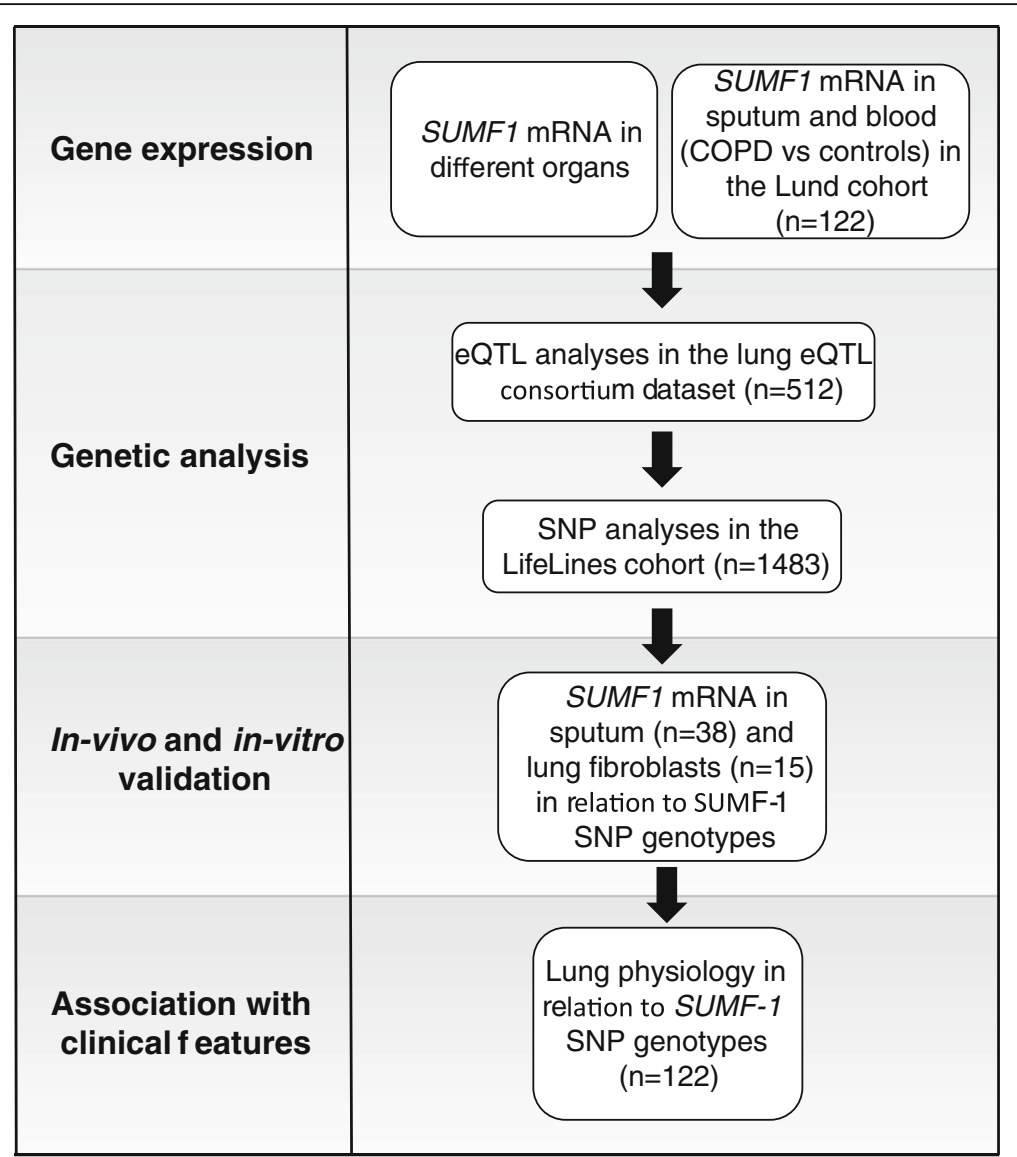

Fig. 1 Flowchart diagram providing an overview of the analyses of SUMF1 in relation to COPD done in this study

Table 1 Characteristics of the total Lund cohort

\begin{tabular}{|c|c|c|}
\hline & $\begin{array}{l}\text { Controls } \\
(n=40)\end{array}$ & $\begin{array}{l}\text { COPD } \\
(n=82)\end{array}$ \\
\hline Sex (male/female) & $19 / 21$ & $46 / 36$ \\
\hline Smoking status (current/former) & $7 / 32^{\mathrm{a}}$ & $24 / 58$ \\
\hline Age (years) & $68(66-70)$ & $67(62-69)$ \\
\hline Pack-years & $26(21-36)^{a}$ & $37(27-48)^{* *}$ \\
\hline BMI $\left(\mathrm{kg} / \mathrm{m}^{2}\right)$ & $27(23-28)$ & $26(23-29)$ \\
\hline $\mathrm{FEV}_{1}$ (\%predicted) & $94(90-103)$ & $60(49-72)^{* * *}$ \\
\hline $\mathrm{FEV}_{1} / \mathrm{FVC}$ & $0.77(0.73-0.79)$ & $0.53(0.44-0.62)^{* * *}$ \\
\hline RV (\%predicted) & $117(102-128)$ & $144(116-165)^{* * *}$ \\
\hline TLC (\%predicted) & $106(99-111)$ & $113(102-122)^{*}$ \\
\hline $\mathrm{RV} / \mathrm{TLC}$ & $0.41(0.38-0.46)$ & $0.47(0.42-0.54)^{* * * x}$ \\
\hline VA (\%predicted) & $90(86-99)$ & $86(79-94)^{*}$ \\
\hline $\mathrm{DL}_{\mathrm{CO}}$ (\%predicted) & $76(69-89)$ & $58(48-68)^{* * *}$ \\
\hline DL $L_{C O} N A$ (\%predicted) & $88(78-96)$ & $69(57-82)^{* * *}$ \\
\hline
\end{tabular}

Pulmonary function data is post inhalation of $\beta 2$ agonist $(400 \mu \mathrm{g}$ salbutamol). Data presented as median (interquartile range). Pack years is defined as the equivalent of smoking 1 pack per day for a year $B M I$ body mass index, $R V$ residual volume, $T L C$ total lung capacity, $V A$ alveolar volume, $D L_{c o}$ diffusion lung capacity ${ }^{*} p<0.05 ;{ }^{* *} p<0.01$ and ${ }^{* * *} p<0.001$

amissing data from 1 patient. * depicts significantly different from controls
(TakaraBio-Clonetech, Saint-Germain-en-Laye, France) was utilized.

For mRNA analyses, RNA was extracted from whole blood, sputum cells, and lung fibroblasts. cDNA synthesis and quantitative real-time PCR (qPCR) was performed as described previously [29].

\section{qPCR analysis in the Lund cohort}

Multiple protein coding splice variants have been identified for SUMF1, of which the functional role and tissue specificity remains unknown. We focused on three wellestablished splice variants in SUMF1 (Splice variants 1 (full length), 2 (lacking exon 3) and 3 (lacking exon 8); For primer sequences and NCBI codes see Additional file 1: Table S1) that were predicted at the time of this study. All mRNA expressions were normalized against expression of the reference genes $\beta$-Actin and GAPDH (see Additional file 1: Table S1).

\section{Patient selection in the Lung eQTL dataset}

To assess associations between the SNPs and SUMF1 gene expression in lung tissue (i.e., cis-acting expression (RNA) quantitative trait loci (cis-eQTL) analysis), the 
Lung eQTL consortium was used, including lung tissue samples obtained from patients at three participating sites; University of Groningen (GRN), Laval University (Laval) and University of British Columbia (UBC) [6].

Tissue was obtained from patients that underwent lung resectional surgery. DNA samples were genotyped with Illumina Human1M-Duo BeadChip arrays, and gene expression profiles were obtained using a custom Affymetrix microarray. Gene expression data is available on the Gene Expression Omnibus accession number GSE23546 and platform GPL10379.

Imputed SNP data was available for 1,095 of the 1,111 subjects, covariate data was missing for another 8 subjects. In the current analyses, we included current and ex-smokers $>40$ years with $\geq 5$ pack-years. COPD was defined as an $\mathrm{FEV}_{1} / \mathrm{FVC}$ ratio $<0.7$. Non-COPD control was defined as an $\mathrm{FEV}_{1} / \mathrm{FVC} \geq 0.7$. In case lung tissue samples were derived from healthy donors, no data on $\mathrm{FEV}_{1}$ or $\mathrm{FEV}_{1} / \mathrm{FVC}$ ratio were available. For $\mathrm{FEV}_{1}$ and $\mathrm{FEV}_{1} / \mathrm{FVC}$, pre-bronchodilator values were used when post-bronchodilator values were not available. Subjects with other lung diseases such as asthma, cystic fibrosis or interstitial lung diseases were excluded. The final dataset included 512 subjects. Patients provided written informed consent and the study was approved by the ethics committees of the Institut universitaire de cardiologie et de pneumologie de Québec and the UBC-Providence Health Care Research Institute Ethics Board for Laval and UBC, respectively. The study protocol was consistent with the Research Code of the University Medical Center Groningen and Dutch national ethical and professional guidelines.

First, cohort specific (GRN, Laval and UBC) principal components (PCs) were calculated based on residuals from linear regression models on 2-log transformed gene expression levels (of each probe separately) adjusted for age, gender and smoking status (never/ever/unknown). PCs that explained at least one percent of the total variance were saved and included as covariates in the main analysis, these were 14 PCs for GRN and Laval, and 16 for UBC. Second, in each cohort separately, linear regression analysis was used to test for association between the SNPs and 2-log transformed gene expression levels. SNPs were tested in an additive genetic model and the models were adjusted for disease status, age, gender, smoking status and the cohort specific number of PCs. Finally, SNP effect estimates of the three cohorts were meta-analyzed using fixed effects models with effect estimates weighted by the inverse of the standard errors.

A cis-eQTL was defined as a SNP that was significantly associated with expression levels of a probe (gene) within a $50 \mathrm{~Kb}$ distance of that SNP. We focused on SNPs which overlapped between eQTL imputed database and Cyto Chip 12, the array used to genotype the Lifelines cohort.

\section{Associations between SUMF1 SNPs and COPD in the LifeLines cohort}

Associations between SUMF1 SNPs and COPD was performed in a Dutch general-population based cohort, the LifeLines cohort study [30]. Subjects with complete genotype and phenotype data (existing data [30]) were included when having smoked at least 5 pack-years and if over 50 years of age. COPD was defined as having $\mathrm{FEV}_{1} / \mathrm{FVC}<0.7$ and $\mathrm{FEV}_{1} \%$ predicted $<80$, based on Quanjer et al.[24] with pre-bronchodilator spirometry following European Respiratory Society/American Thorax Society criteria [24]. Controls were defined as having $\mathrm{FEV}_{1} / \mathrm{FVC} \geq 0.7$ and $\mathrm{FEV}_{1} \%$ predicted $>90$.

In the Lifelines cohort, genotyping was performed using IlluminaCytoSNP-12 arrays and SNPs were included that fulfilled the quality control criteria: genotype call-rate $\geq 95 \%$, minor allele frequency $\geq 1 \%$, and Hardy-Weinberg equilibrium cut-off $p$-value $\geq 10^{-4}$. Samples with call rates below $95 \%$ were excluded.

\section{SUMF1 genotyping in the Lund cohort}

Whole blood was taken from all subjects in the Lund cohort and DNA was extracted. All patients were genotyped for the SUMF1 SNPs identified to be top hits in the eQTL analysis and Lifelines using Agena iPLEX genotyping. Genotyping was performed at the Mutation Analysis Facility at Karolinska University Hospital (Huddinge, Sweden) using iPLEX $^{\circ}$ Gold chemistry and MassARRAY ${ }^{\circ}$ mass spectrometry system [31] (Agena Bioscience, San Diego, CA, U.S.A.). Multiplexed assays were designed using MassARRAY ${ }^{\circ}$ Assay Design v4.0 Software (Agena Bioscience). Protocol for allele-specific base extension was performed according to Agena Bioscience's recommendation. Analytes were spotted onto a 384-element SpectroCHIP II array (Agena Bioscience) using Nanodispenser RS1000 (Agena Bioscience) and subsequently analyzed by MALDI-TOF on a MassARRAY ${ }^{\bullet}$ Compact mass spectrometer (Agena Bioscience). Genotype calls were manually checked by two persons individually using MassARRAY ${ }^{\circ}$ TYPER v4.0 Software (Agena Bioscience).

\section{Statistics}

Descriptive statistics are presented as median (interquartile range $(\mathrm{IQR})) . P<0.05$ was considered significant.

The differences in gene expression in sputum and blood between controls and COPD patients were analyzed using the Mann-Whitney $U$-test using GraphPad Prism 5 (Graphpad, La Jolla, CA, USA). In the Lund cohort the associations between SNPs and gene expression in sputum and lung fibroblasts as well as in the lung physiology was tested using the Kruskal-Wallis test including Dunn's Multiple Comparison Post Test (using Graph Pad Prism 5 software). The eQTL-analyses using the lung eQTL-consortium data are described above. 
The association between SNPs and COPD in the Dutch cohort was performed using logistic regression models including the SNP in a co-dominant genetic model and adjusted for sex, age, and pack years using SPSS version 22

Finally, associations between the SNPs (in an additive model) and lung function parameters were tested using linear regression adjusted for COPD, smoking status, and age in the Lund cohort (using SPSS version 22).

\section{Results}

\section{Description of the Lund cohort}

Table 1 shows the descriptive statistics of the Lund cohort. An adequate sputum sample, from which RNA could be extracted, could be obtained from 38 subjects (19 controls and 19 COPD patients, Additional file 1: Table S2) in the Lund cohort. Additional file 1: Table S3 shows the descriptive statistics of the 15 COPD patients in the Lund cohort that performed a bronchoscopy, and from which lung fibroblasts were obtained.

\section{SUMF1 expression is altered in COPD patients compared to controls}

We found that SUMF1 mRNA was expressed relatively high in whole lung tissue (Figs. 2a-d), and specifically, Splice variant 3 showed the highest expression in lung tissue compared to all other investigated tissues in the body (Fig. 2d).

To examine if SUMF1 expression was systemic or lung specific, sputum cells and whole blood from COPD patients and controls from the Lund cohort were examined for differences in SUMF1 expression. In sputum cells (Figs. 2e-h), all three splice variants examined were detectable and showed significantly lower levels in COPD patients than controls in Splice variant $2(p=0.018)$ and Splice variant $3(p=0.0086)$. While in contrast, in whole blood there was no significant difference in total SUMF1 expression between controls and COPD patients $(p=0.39$, Additional file 2: Figure S1), and the three splice variants were unable to be detected in the majority of individuals.

\section{Lung expression quantitative trait loci (eQTL) analysis and linkage disequilibrium analysis}

We next performed an expression quantitative trait loci (eQTL) analysis in lung tissue in order to determine whether the differential gene expression of SUMF1 were associated with genetic polymorphisms. In the three large cohorts (Groningen, Laval, and UBC; $n=512$ ) examined, twelve of the SNPs, that overlapped with the array used to genotype the Lifelines cohort, showed significant expression differences (Table 2).

A linkage disequilibrium (LD) analysis (HaploView 4.2) show the associations between the twelve SUMF1 SNPs identified (Fig. 3a).
The top hit SNP from the eQTL analysis, rs11915920 (Fig. 4a) provided a strong eQTLs (Table 2). For further data presentation in this study, the most significant SNP associated with gene expression, i.e., rs11915920, is used for further data presentation in this study.

The SNP rs793391 (Fig. 4b), the most significant SNP from the Lifelines cohort (see below), was also a significant eQTL (Table 2), but to a much smaller extent.

\section{SUMF1 SNPs show differences in SUMF1 expression in the lung}

In the Lund cohort, the SUMF1 mRNA levels, of total SUMF1 and the different splice variants, were examined in sputum cells from controls and COPD patients as well as in lung fibroblasts from COPD patients in relation to the SUMF1 genotypes of SNPs rs11915920 and rs793391.

Similar trends in SUMF1 mRNA expression were seen in both sputum cells and fibroblasts with SNP rs11915920 (Fig. 5). Significant differences were observed among the rs11915920 genotypes, with a higher expression level in subjects homozygous for the reference allele $(C)$, in all splice variants in sputum cells (Fig. 5b-d; Splice variant 1: $p=0.017$, Splice variant 2: $p=0.038$, Splice variant 3: $p=0.015)$. In lung fibroblasts, the expression of Splice variant 3 was significantly different between the genotypes (Fig. 5h, $p=0.014$ ). These in vitro findings validate the eQTL analysis where there were also higher levels of mRNA expression observed in subjects with the reference allele (C) of rs11915920 (Fig. 4a). The top candidate from our SNP analyses of the Lifelines cohort (see below), rs793391, did not show any association with SUMF1 expression in sputum cells or lung fibroblasts (Additional file 2: Figure S2). rs793391 was a much weaker candidate than rs11915920 in the eQTL analysis and the in vitro analysis corroborates these results.

\section{Association between SUMF1 SNPs and COPD}

We also investigated the association between the SUMF1 SNPs associated with eQTLs and COPD in the Dutch cohort, LifeLines $(n=1483$, for descriptive statistics see (Additional file 1: Table S4)). Convincingly, the reference allele (A) of SNP rs793391 was associated with a higher risk for COPD in the LifeLines cohort (Table 3). In addition, the SNP rs308739 was also associated with COPD. (For allele frequencies, see Table 4). The most significant SNP from the association between SUMF1 and COPD, rs793391, was chosen to be followed up pathophysiologically in this study.

\section{SNP in SUMF1 is associated with lung function}

When examining advanced lung physiology in subjects from the Lund Cohort, including controls and COPD patients, we found that among the rs793391 genotypes there was an overall difference in $\mathrm{FEV}_{1} / \mathrm{FVC}(p=0.031)$, 

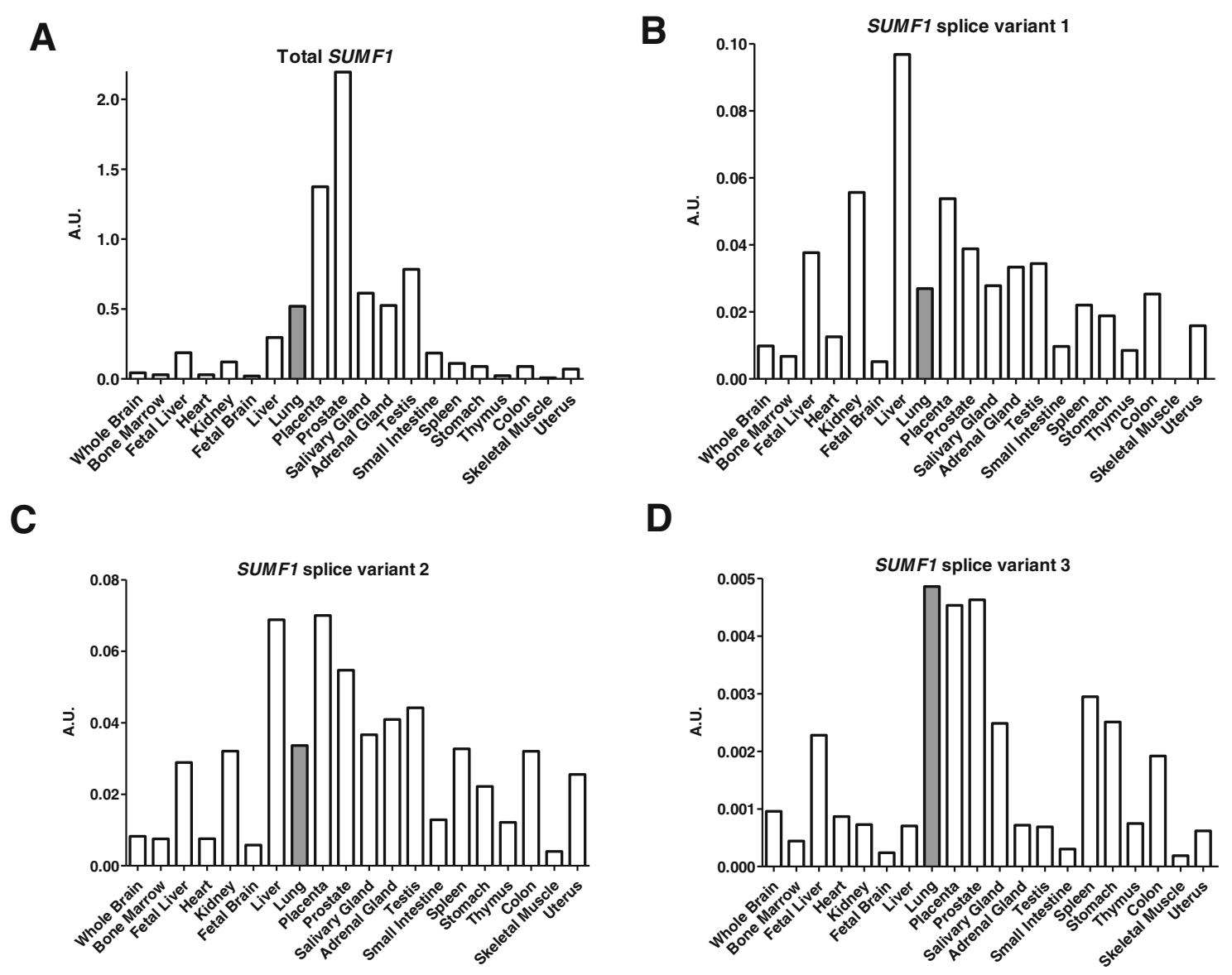

E

$\mathbf{F}$
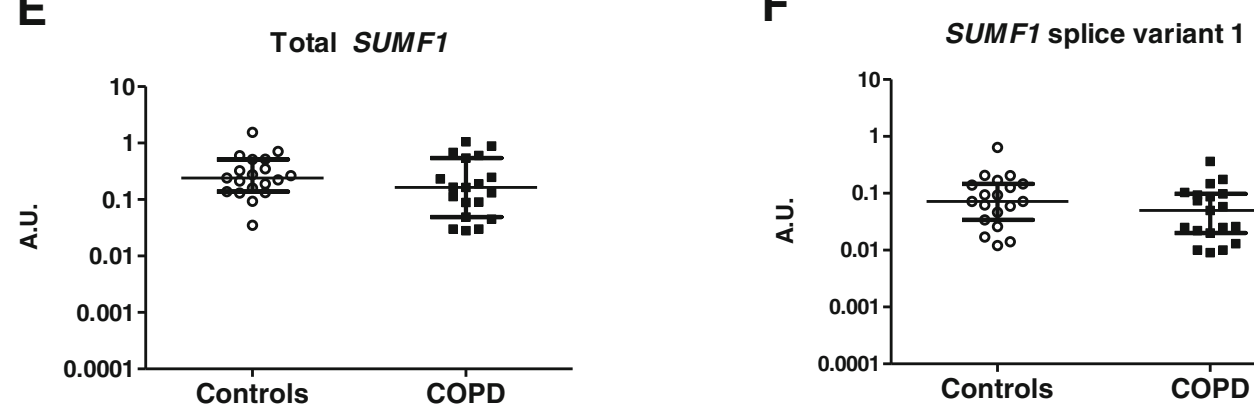

G

SUMF1 splice variant 2

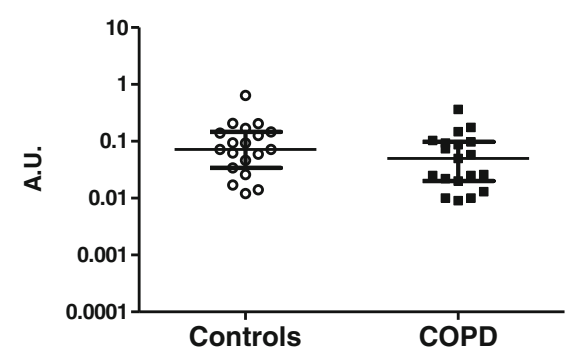

H

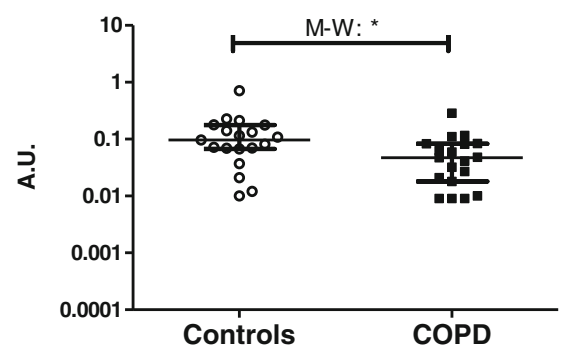

Fig. 2 (See legend on next page.) 
(See figure on previous page.)

Fig. 2 SUMF1 expression is altered in COPD patients. A master panel of mRNA from twenty different human tissues was probed for total SUMF1 mRNA expression (a) as well as three individual Splice variants 1 (b), 2 (c) and 3 (d). Total SUMF1 mRNA expression (e) as well as Splice variant 1 (f), 2 (g) and 3 (h) expression were examined in sputum cells from COPD patients and controls in the Lund cohort. ${ }^{*}=p<0.05$ and ${ }^{* *}=p<0.01$, A.U. = Arbitrary units, M-W = Mann-Whitney test used

$\mathrm{FEV}_{1} \%$ predicted $(p=0.035)$, diffusion capacity $\left(D_{\mathrm{LCO}}=\right.$ lung diffusion capacity for carbon monoxide)\%predicted $(p=0.027)$ and alveolar volume (VA)\%predicted ( $p=$ 0.040). Specifically, subjects homozygous for the reference allele of rs793391 had lower $\mathrm{FEV}_{1} / \mathrm{FVC}$ and $\mathrm{FEV}_{1}$ \%predicted compared to heterozygous subjects (Fig. 6a and b, respectively). A similar pattern was seen in $D_{\mathrm{LCO}} \%$ predicted (Fig. 6c) and VA\%predicted among the different SUMF1 rs793391 genotypes, but not in $D_{\mathrm{LCO}} / \mathrm{VA} \%$ predicted. Interestingly, even after correction for COPD, smoking status and age, the association between rs793391 and $D_{\mathrm{LCO}} \%$ predicted remained significant, while the association between rs793391 and $\mathrm{FEV}_{1}$ / $\mathrm{FVC}, \mathrm{FEV}_{1} \%$ predicted and VA\%predicted did not (Additional file 1: Table S5).

Neither residual volume, total lung capacity, nor air trapping index (residual volume/total lung capacity) showed any difference among the different genotypes of rs793391 (data not shown).

The SNP rs11915920, highly significant in the eQTL analyses, did not have any significant association with measured lung function parameters (Additional file 2: Figure S3), neither had the SNP rs308739.

\section{Discussion}

We found that SUMF1 was associated with COPD. Primarily we showed that SUMF1 is differently expressed in sputum cells from COPD patients and controls. In addition, eQTL analysis revealed that several SNPs were significantly associated with SUMF1 expression, with the top hit being SNP rs11915920. This was further verified in mRNA from sputum cells and lung fibroblasts, and the main differences were in SUMF1 Splice variant 3. We also show that two SNPs in SUMF1, rs793391 and 308739 , were associated with increased risk of COPD in a population based cohort, LifeLines. Finally, we found that rs793391 was associated with differences in lung function parameters.

Our study found, that SUMF1 Splice variant 3 was most highly expressed in whole lung tissue as compared to other tissues examined in the body and showed the biggest expression effect in lung fibroblasts. Splice variant 3 lacks exon 8 in SUMF1 (Fig. 3b) but, currently, no effects regarding the protein function or structure of this variant have been reported. Additionally, rs11915920, the top hit SNP related to SUMF1 expression in lung tissue, is in close proximity to SUMF1 exon 8 (Fig. 3b), and might affect the splicing of exon 8. Perhaps Splice variant 3 is an important variant of SUMF1 specifically in the lungs with a yet unknown function. Future studies will be needed to investigate this possibility.

The importance of SUMF1 to the development and maintenance of alveoli was recently discovered in mice $[21,22]$. Although Sumf1 -/- mice have a very short

Table 2 eQTL analysis of SUMF1 SNPs in lung tissue from three large cohorts (Groningen, Laval, and UBC; $n=512$ )

\begin{tabular}{lllccc}
\hline SNP & Ref & Var & eQTL meta-estimate (B) & eQTL meta-standard error (SE) & eQTL meta- $p$-value \\
\hline rs11915920 & C & T & -0.110 & 0.009 & $\mathbf{6 . 4 1 E - 3 8}$ \\
rs2819562 & C & T & -0.096 & 0.009 & $\mathbf{2 . 4 6 E - 2 6}$ \\
rs809437 & A & G & -0.081 & 0.011 & $\mathbf{2 . 4 1 E - 1 4}$ \\
rs17030493 & T & C & 0.066 & 0.013 & $\mathbf{3 . 6 4 E - 0 7}$ \\
rs1687863 & G & A & 0.056 & 0.013 & $\mathbf{6 . 9 7 E - 0 6}$ \\
rs1968930 & A & C & 0.054 & 0.014 & $\mathbf{7 . 8 4 E - 0 5}$ \\
rs1688411 & T & G & 0.048 & 0.014 & $\mathbf{0 . 0 0 0 5}$ \\
rs807785 & C & T & 0.037 & 0.011 & $\mathbf{0 . 0 0 1 1}$ \\
rs308739 & A & C & -0.060 & 0.019 & $\mathbf{0 . 0 0 1 9}$ \\
rs1688413 & C & T & 0.035 & 0.012 & $\mathbf{0 . 0 0 2 8}$ \\
rs17040589 & C & T & -0.050 & 0.021 & $\mathbf{0 . 0 1 9 9}$ \\
rs793391 & A & C & 0.022 & 0.011 & $\mathbf{0 . 0 4 0 0}$ \\
\hline
\end{tabular}

Presented are SNPs that were significantly associated with expression levels of a probe (gene) within a 50Kb distance of that SNP and overlapped with the array used to genotype the Lifelines cohort. Bold indicates significant values

Ref reference allele, Var variance allele 

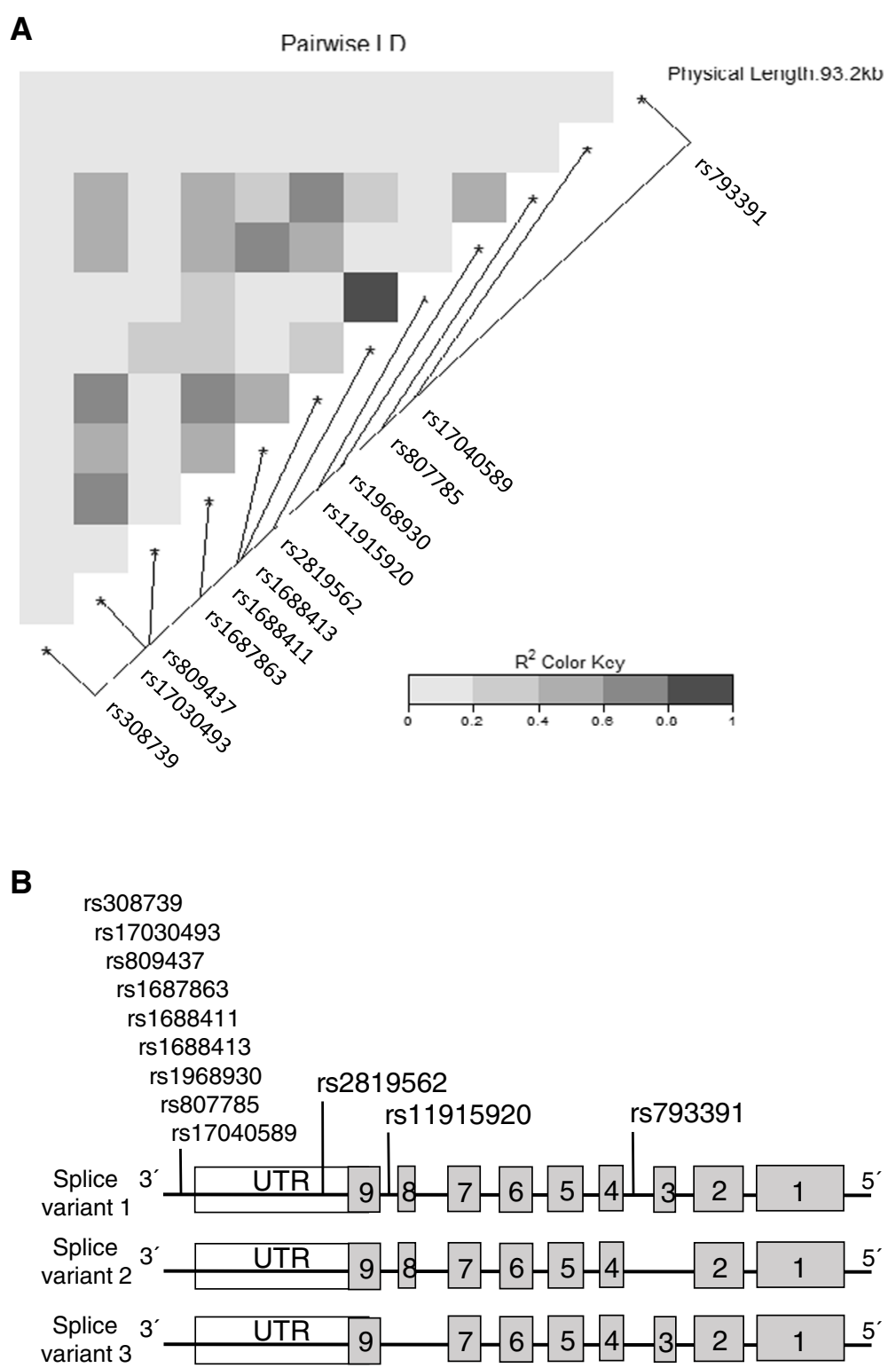

Fig. 3 Linkage Disequilibrium analysis of SUMF1 SNPs. An LD plot (a) shows the 12 SUMF1 SNPs overlapping between the eQTL analysis and the array used to genotype the Lifelines cohort. A schematic picture (b) showing localization of the 12 SNPs on the SUMF1 mRNA, and the different splice variants. Boxes showing exon 1-9, UTR = untranslated region

lifespan, they have provided a wealth of information regarding sulfatase activation and function. In these mice, there was an overabundance of sulfated GAGs resulting in inactive sulfatases, leading to an arrest in the alveolarization process and an emphysema-like phenotype [22]. This emphysema-like phenotype was one of our first hints that perhaps SUMF1 may play a role in the development of COPD, which is hallmarked by the development of emphysema. In addition to the emphysema-like phenotype, many cell and tissue types were found to have massive GAG accumulation in the Sumf1 -/- mice, but this has not yet been investigated in COPD.
We show that $\mathrm{D}_{\mathrm{LCO}}$ \%predicted is independently affected by the rs793391, since it is not driven by the disease or smoking status, which is the case for $\mathrm{FEV}_{1} / \mathrm{FVC}$ and $\mathrm{FEV}_{1}$ \%predicted (Additional file 1: Table S5). Our finding that $\mathrm{D}_{\mathrm{LCO}}$ \%predicted is lower in patients with the reference allele (A) of rs793391 is in accordance with the Sumf1 -/- mouse showing a deficient alveolar septation and a subsequent arrest in alveolar formation. Interestingly, no difference in residual volume, air trapping index (residual volume/total lung capacity), or $\mathrm{D}_{\mathrm{LCO}}$ \%predicted corrected for alveolar volume $\left(\mathrm{D}_{\mathrm{LCO}} /\right.$ VA\%predicted) was observed between the SUMF1 

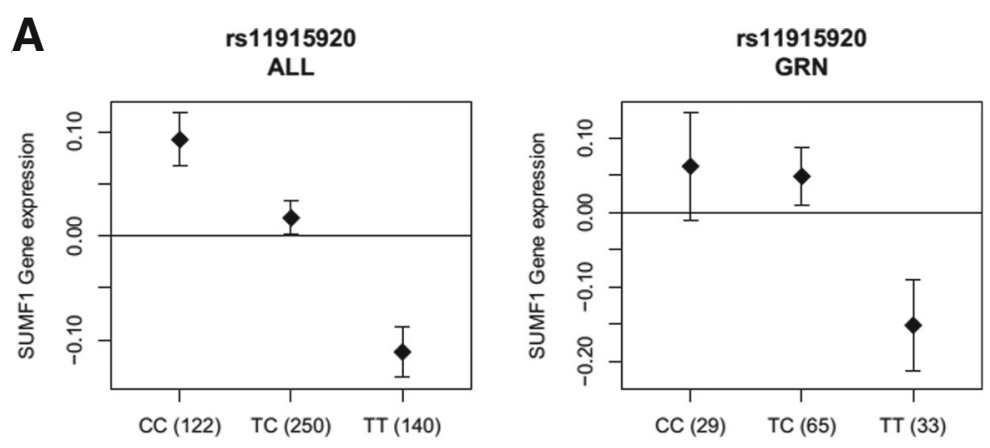

MEST $=-0.11 \mathrm{MSE}=0.01 \mathrm{MP}=4.34 \mathrm{e}-40$

$E S T=-0.13 \mathrm{SE}=0.03 \mathrm{P}=1.76 \mathrm{e}-06$
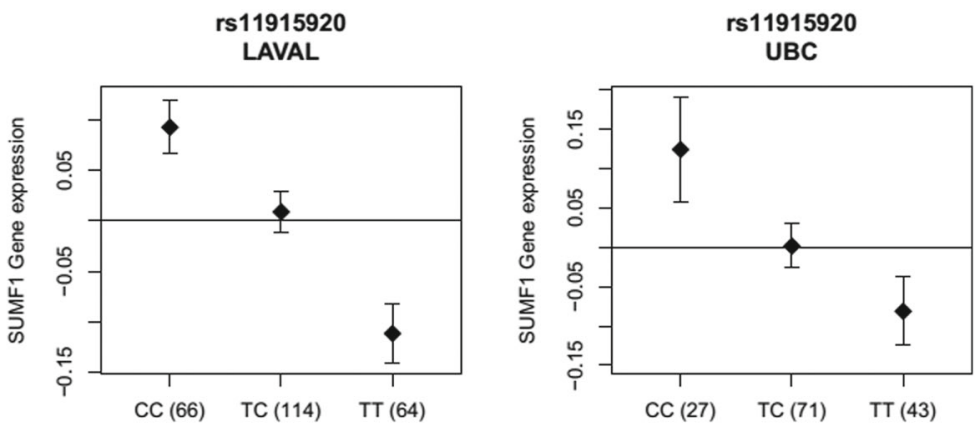

$E S T=-0.11 \mathrm{SE}=0.01 \mathrm{P}=4.53 \mathrm{e}-21$

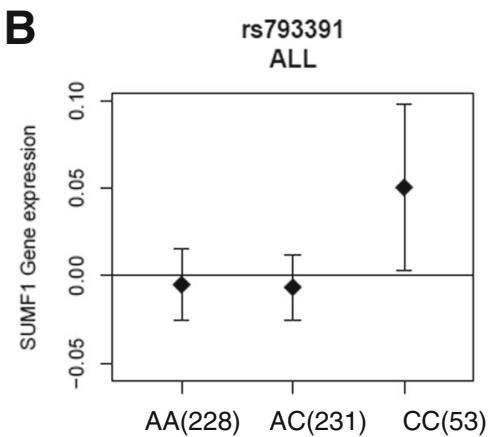

$E S T=-0.12 \mathrm{SE}=0.02 \mathrm{P}=2.31 \mathrm{e}-09$

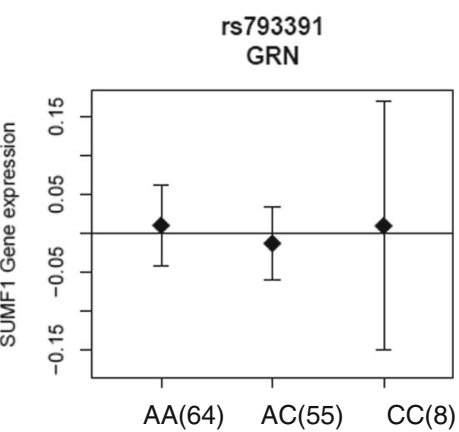

MEST $=0.02 \mathrm{MSE}=0.01 \mathrm{MP}=0.0348$

$\mathrm{EST}=-0.01 \mathrm{SE}=0.03 \mathrm{P}=0.664$
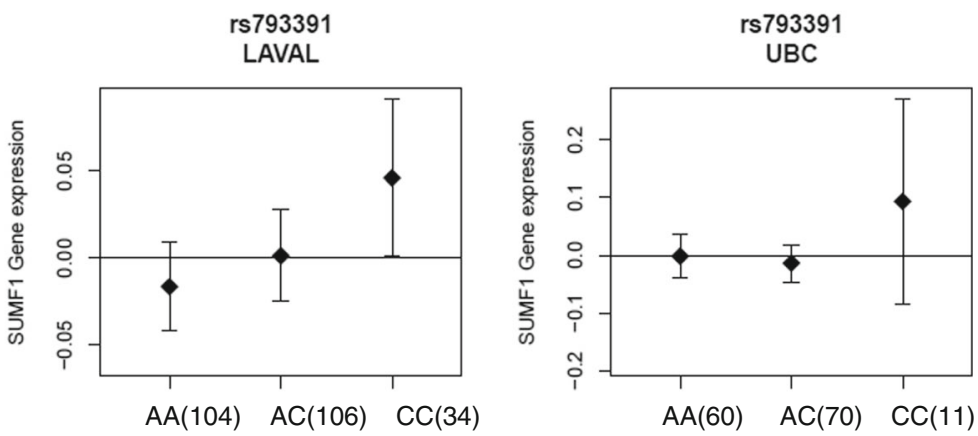

$\mathrm{EST}=0.03 \mathrm{SE}=0.01 \mathrm{P}=0.0255$

$\mathrm{EST}=0.02 \mathrm{SE}=0.02 \mathrm{P}=0.352$

Fig. 4 eQTL analysis of SUMF1 SNPs. Each set of box plots represents the three different cohorts, combined (ALL) as well as separately ( $G R N=$ Groningen, Laval = Laval University and UBC = University of British Columbia), and the corrected expression differences seen between the different SNP genotypes. a represents the SNP rS11915920 and b represents the SNP rs793391. Genotype is presented with the reference/reference genotype to the left 


\section{SNP rs11915920}

A

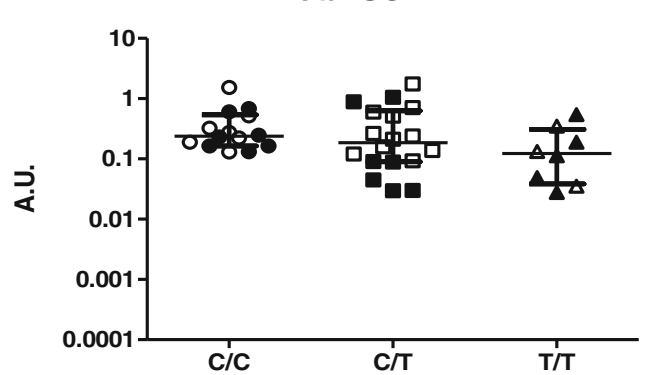

C

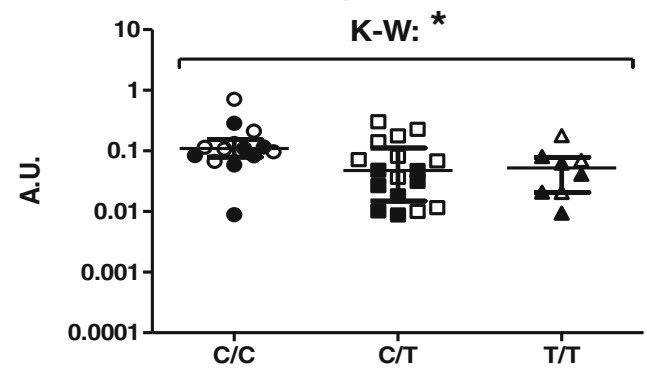

E
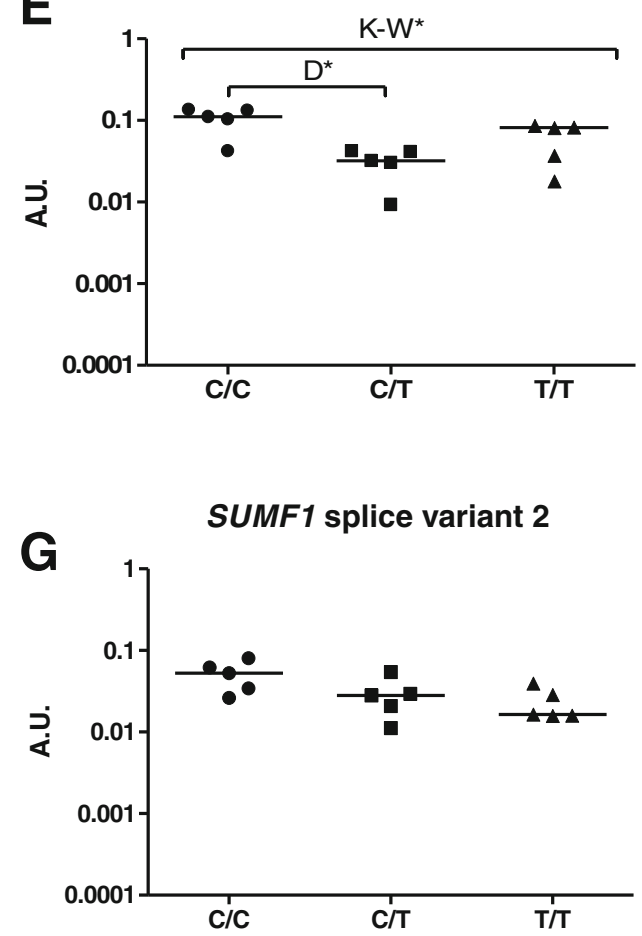

B
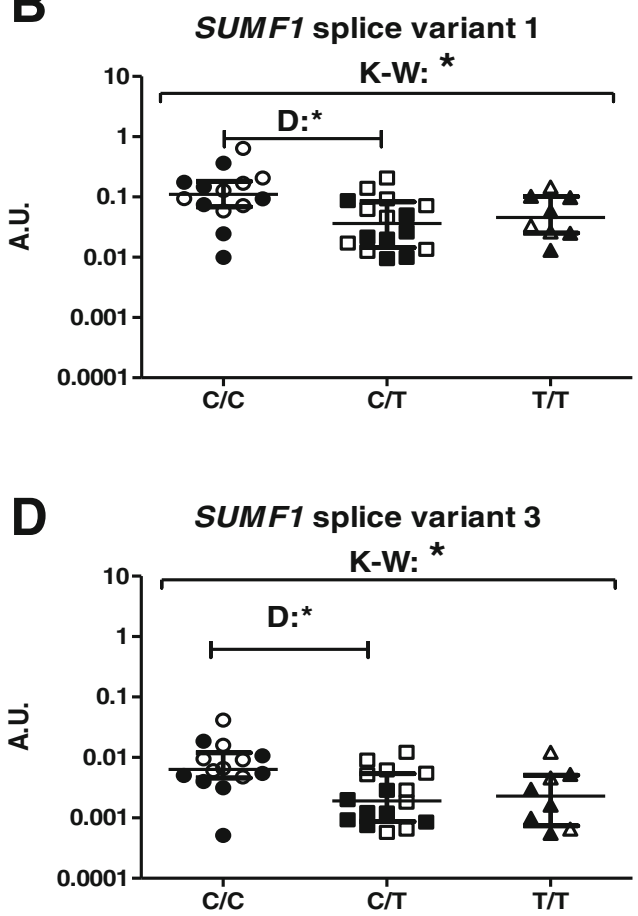

$\mathbf{F}$
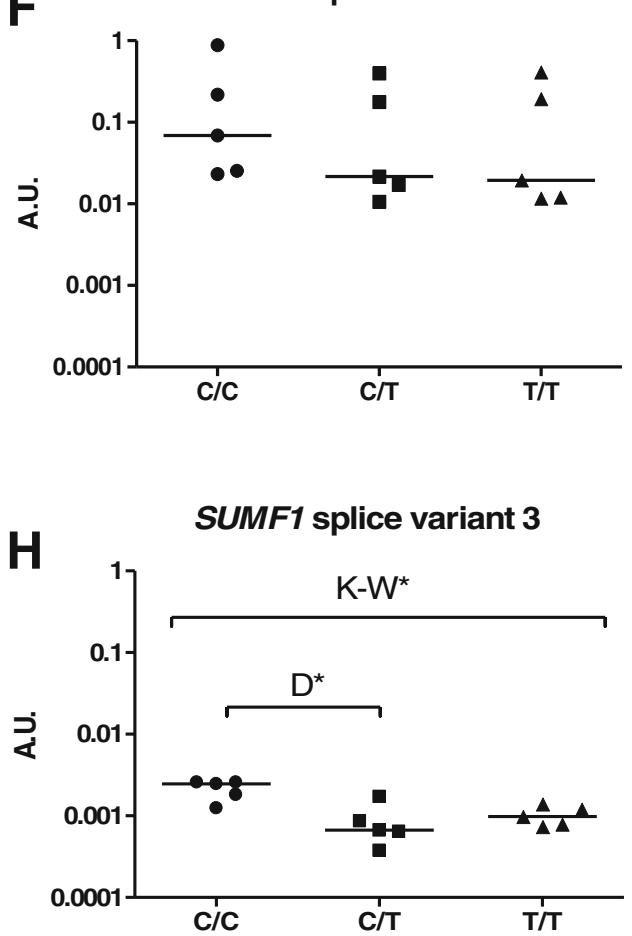

Fig. 5 (See legend on next page.) 
(See figure on previous page.)

Fig. 5 SUMF1 expression in sputum cells and lung fibroblasts divided by rs11915920 genotype. SUMF1 expression, including the three splice variants, was examined for SNP rs 11915920 in sputum cells (a-d) and lung fibroblasts (e-h) from subjects from the Lund cohort. Both controls and COPD patients were used for sputum cell analysis and COPD patients for the lung fibroblasts, then divided depending on genotype. Open symbols $=$ controls, filled symbols $=$ COPD patients. A.U. = Arbitrary units, ${ }^{*}=$ significance at $p<0.05 . \mathrm{K}-\mathrm{W}=$ Kruskal-Wallis test was used, followed by Dunn's multiple comparison post tests $(=D)$. Genotype is presented with the reference/reference genotype to the left

genotypes. These findings are also in agreement with the Sumf1 -/- mouse, suggesting a developmental perturbation of distal alveolar septation rather than a destructive process. Future studies will be performed in order to focus on extensive lung physiology in larger cohorts to determine if the clinical phenotype related to SUMF1 SNPs holds true.

In lung function data, the subjects with homozygous reference genotype of rs793391 (AA) showed impaired lung physiology compared to the respective heterozygous genotype. The heterozygous genotype thereby appears to be protective in association with COPD. However, there was no significant differences between the homozygous reference and variance genotypes (AA versus $C C$ in rs793391), which might be due to the low number of patients in the Lund cohort that had the homozygous variance genotype. Unfortunately, only flow-volume spirometry was performed in the Lifelines cohort, so we were not able to verify the differences in

Table 3 Logistic regression models assessing associations between SUMF1 SNPS and COPD (additive model) in the LifeLines cohort

\begin{tabular}{|c|c|c|c|c|c|}
\hline \multirow[b]{2}{*}{ SUMF1 SNP } & \multirow[b]{2}{*}{ Ref } & \multirow[b]{2}{*}{ Var } & \multicolumn{3}{|c|}{$\begin{array}{l}\text { LifeLines cohort } \\
n=1483\end{array}$} \\
\hline & & & OR & SE & $p$-value \\
\hline rs793391 & A & $C$ & 1.42 & 0.13 & 0.0066 \\
\hline rs308739 & A & $C$ & 0.40 & 0.36 & 0.010 \\
\hline rs807785 & $C$ & $\mathrm{~T}$ & 0.82 & 0.13 & 0.14 \\
\hline rs1688411 & T & G & 0.77 & 0.18 & 0.16 \\
\hline rs1968930 & $A$ & $C$ & 0.78 & 0.19 & 0.19 \\
\hline rs1687863 & G & A & 0.84 & 0.15 & 0.24 \\
\hline rs17030493 & T & $C$ & 0.87 & 0.17 & 0.39 \\
\hline rs1688413 & C & $\mathrm{T}$ & 0.90 & 0.14 & 0.44 \\
\hline rs809437 & $A$ & G & 0.92 & 0.13 & 0.55 \\
\hline rs17040589 & C & $\mathrm{T}$ & 0.88 & 0.25 & 0.62 \\
\hline rs11915920 & $C$ & $\mathrm{~T}$ & 0.99 & 0.12 & 0.90 \\
\hline rs2819562 & C & $\mathrm{T}$ & 1.01 & 0.12 & 0.96 \\
\hline
\end{tabular}

Shown are SNPs that were significant eQTLs and overlapped with the array used to genotype the Lifelines cohort. $\mathrm{OR}=$ odds ratio, $\mathrm{SE}=$ standard error, $p$-value is from logistic regression models assessing associations between SNPs (additive model) and COPD, adjusted for sex, age, and pack years. Smoking controls were defined as an $\mathrm{FEV}_{1} / \mathrm{FVC}>0.7$ and COPD was defined as an $\mathrm{FEV}_{1}$ / $\mathrm{FVC}<0.7$. Ref $=$ reference allele. Var $=$ variance allele. Bold indicates significant values
$D_{\text {LCO }} \%$ predicted observed in patients with various rs793391 genotypes in the Lund cohort. Future studies will be needed to determine if this potentially protective genotype holds true for other populations.

A recent GWAS identified genetic variants associated with total lung capacity in COPD [4]. Among several SNPs that were identified in patients with prominent emphysema, one was in SUMF1, however, it was not studied futher. This GWAS identified SNP was neither present in our analysis platforms, nor was it found to be in LD with either of SNPs described in our study.

To our knowledge this is the first study to genetically focus on SUMF1 in the context of COPD. Our results indicate that the different SUMF1 SNPs may be responsible for different factors in the development of the disease. We showed that several SNPs were associated with SUMF1 expression, however, on a functional level the molecular mechanism and their relationship to COPD remains undiscovered. Alternatively, as all of the SUMF1 SNPs from this study were found to be in introns or untranslated regions (none are found in translated exons), there is the possibility that they may act as small RNA precursors, such as microRNAs. These small RNAs may, in turn, regulate the expression of SUMF1 or another unknown gene, but this possibility has yet to be

Table 4 Genotype and allele frequencies in the LifeLines cohort

\begin{tabular}{|c|c|c|c|c|c|c|}
\hline \multirow[b]{2}{*}{ SUMF1 SNP } & \multicolumn{6}{|c|}{$\begin{array}{l}\text { LifeLines cohort } \\
n=1483\end{array}$} \\
\hline & Ref & Var & $\begin{array}{l}\text { Ref/Ref } \\
\text { genotype n (\%) }\end{array}$ & $\begin{array}{l}\text { Ref/Nar } \\
\text { genotype n (\%) }\end{array}$ & $\begin{array}{l}\text { VarNar } \\
\text { genotype n (\%) }\end{array}$ & MAF \\
\hline rs793391 & A & $C$ & 669 (45) & $644(43)$ & $170(12)$ & 0.33 \\
\hline rs308739 & A & $C$ & $6(0.4)$ & $137(9)$ & $1340(90)$ & 0.05 \\
\hline rs807785 & C & $\mathrm{T}$ & $122(8)$ & $587(40)$ & 774 (52) & 0.28 \\
\hline rs1688411 & $\mathrm{T}$ & G & $24(2)$ & 356 (24) & $1103(74)$ & 0.14 \\
\hline rs1968930 & A & $C$ & $22(2)$ & 339 (23) & $1122(76)$ & 0.13 \\
\hline rs1687863 & G & A & $54(4)$ & 455 (31) & 974 (66) & 0.19 \\
\hline rs17030493 & $\mathrm{T}$ & C & $35(2)$ & 395 (27) & $1053(71)$ & 0.16 \\
\hline rs1688413 & C & $\mathrm{T}$ & $95(6)$ & $527(36)$ & 861 (58) & 0.24 \\
\hline rs809437 & A & G & $103(7)$ & $597(40)$ & 783 (53) & 0.27 \\
\hline rs17040589 & $C$ & $\mathrm{~T}$ & $9(1)$ & $164(11)$ & $1310(88)$ & 0.06 \\
\hline rs11915920 & $C$ & $\mathrm{~T}$ & $366(25)$ & 766 (52) & 351 (24) & 0.49 \\
\hline rs2819562 & $C$ & $\mathrm{~T}$ & $293(20)$ & 775 (52) & 415 (28) & 0.46 \\
\hline
\end{tabular}

Ref reference allele, Var variance allele, MAF minor allele frequency 


\section{SNP rs793391}
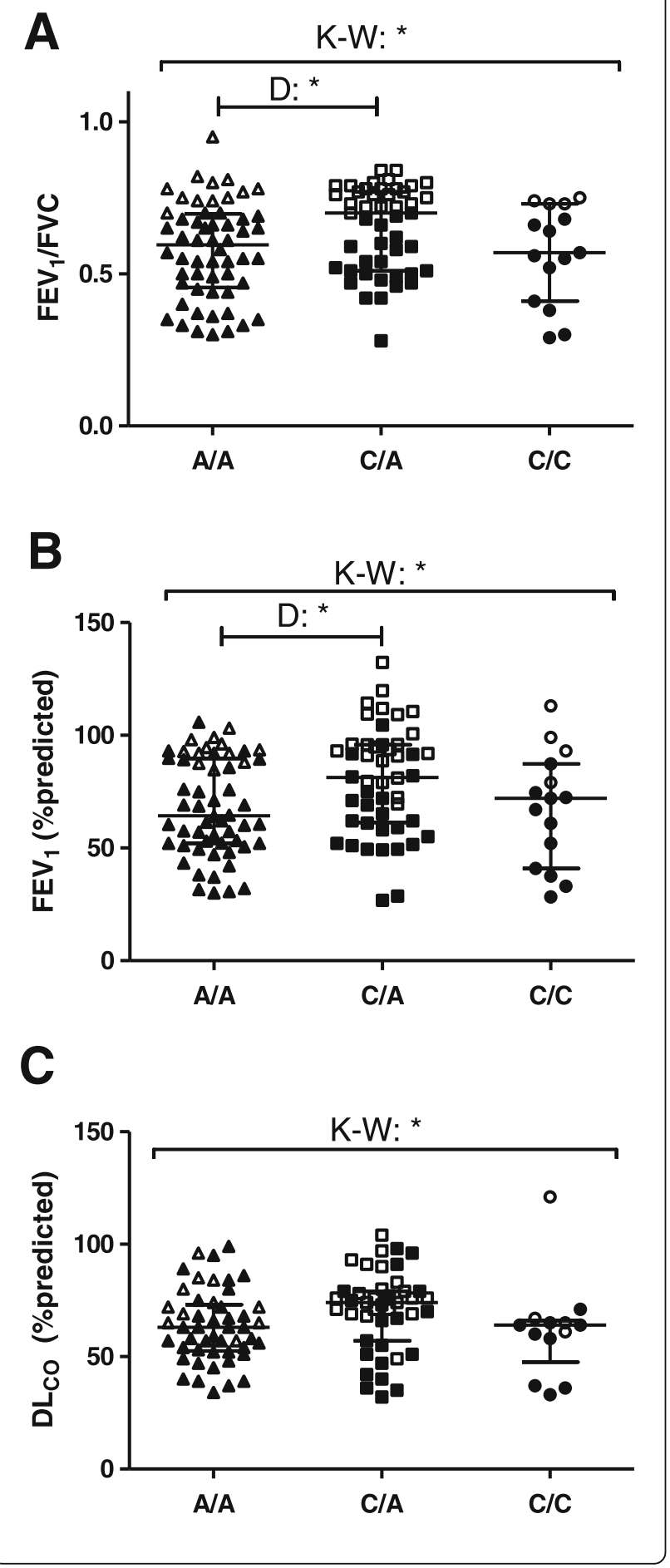

Fig. 6 Lung function in COPD patients and controls divided by SNP rs793391 genotypes. FEV 1 /FVC (a), FEV (b) and DL $_{\text {co }}$ \%predicted (c) of subjects from the Lund cohort are divided according to the genotype of rs793391. Open symbols $=$ controls, filled symbols $=$ COPD patients. ${ }^{*}=p<0.05,{ }^{*}=p<0.01 . \mathrm{K}-\mathrm{W}=$ Kruskal-Wallis test was used, followed by Dunn's multiple comparison post tests (=D). Genotype is presented with the reference/reference genotype to the left

examined. In contrast to rs11915920, which was strongly associated to SUMF1 expression, rs793391 had a uniform impact on lung function. These findings lead us to believe that the different SNPs may have different roles in the biology of the disease. SUMF1 is a good candidate for further study into how the genotype of patients affects the different phenotypes of COPD on a molecular level. Future studies into downstream effects of SUMF1, such as sulfatase activity would need to be undertaken and we can begin to delve deeper into the molecular mechanisms of the disease and work towards better possible treatments for those affected.

A limitation of the study is that the different cohorts have been analysed with platforms investigating different SNPs, and subsequently only twelve of the significant SNPs in the lung tissue dataset were found in the Lifelines cohort. Another limitation is the difference in rationale for inclusion in the cohorts. The LifeLines cohort is a large general-population based study, giving a high power. However, most COPD patients have only a mild disease, and the possibility of finding relevant genes in a multigenetic disease such as COPD might then be difficult. This might explain why there is a lack of association between COPD and several of the different SNPs. This could also explain why there is a strong relationship between rs11915920 and SUMF1 expression, but no direct association to COPD in the population based LifeLines cohort. Maybe a cohort including patients with more severe COPD would give a significant association between rs11915920 and COPD. This is suggested from a subanalysis of the Lund cohort, comparing 24 more severe COPD patients versus the contrasting 24 clearly healthy controls, showing a significant association to rs11915920 (data not shown), even though the subject numbers were low. This hypothesis needs to be further explored in larger cohorts where more patients with severe COPD are included.

\section{Conclusion}

We provide evidence that expression and genetic regulation of SUMF1 differs between smokers with and without COPD. SUMF1 is differentially expressed in sputum cells from COPD patients and controls. Through examination of the SUMF1 gene, we found SNPs that 
significantly affect mRNA levels through the use of an eQTL analysis from a lung tissue dataset, which was corroborated in vitro by mRNA expression analysis of sputum cells and lung fibroblasts from the Lund cohort. In addition, some of these SNPs in SUMF1 are associated with an increased risk of COPD. Furthermore, the different SUMF1 SNPs were found to have differential effects in COPD. Some SNPs, such as rs11915920, had an effect on SUMF1 mRNA expression in tissue, sputum cells, and lung fibroblasts, while the SNP rs793391 was significantly associated with lung function parameters and thereby COPD.

\section{Additional files}

Additional file 1: Table S1. Primer sequences used for GPCR. Table S2. Characteristics of controls and COPD patients who could expectorate an adequate sputum. Table S3. Characteristics of COPD patients from whom lung fibroblasts were obtained. Table $\mathbf{S 4}$. Characteristics of subjects included in the LifeLines cohort. Table S5. Associations between SNP rs793391 and $\mathrm{FEV}_{1} / \mathrm{FVC}$, FEV $\%$ predicted and DL $\mathrm{L}_{\mathrm{C}} \%$ predicted using linear regression models adjusted for current smoking status, age, and COPD. (DOCX $18 \mathrm{~kb})$

Additional file 2: Figure S1. SUMF1 expression in blood from COPD patients and controls. Total SUMF1 mRNA expression was examined in whole blood from COPD patients and controls in the Lund cohort. A.U. = Arbitrary units. Figure S2. SUMF1 expression in sputum cells and lung fibroblasts divided by rs793391 genotypes. SUMF1 expression, including the three splice variants, was examined for the SNP rs793391 genotype in sputum cells (A-D) from controls and COPD patients and in lung fibroblasts (E-H) obtained from COPD patients from the Lund cohort. Open symbols = controls, filled symbols = COPD patients, A.U. = Arbitrary units. Genotype is presented with the reference/reference genotype to the left. Figure S3. Lung function in COPD patients and controls divided by SNP rs11915920 genotype. FEV $/$ /FVC (A), FEV $(B)$ and DLCO \%predicted (C) of subjects from the Lund cohort are divided according to the genotype of rs11915920. Open symbols $=$ controls, filled symbols = COPD patients. Genotype is presented with the reference/ reference genotype to the left. (PPTX $275 \mathrm{~kb}$ )

\section{Abbreviations}

COPD: Chronic obstructive pulmonary disease; $\mathrm{DL}_{\text {co: }}$ L Lung diffusion capacity for carbon monoxide); eQTL: Expression (RNA) quantitative trait loci; $\mathrm{FEV}_{1}$ : Forced expiratory volume in 1 second; FVC: Forced volume capacity; GAGs: Glycosaminoglycans; GWAS: Genome-wide association studies; SNP: Single nucleotide polymorphism; SUMF1: Sulfatase modifying factor 1; VA: Alveolar volume

\section{Acknowledgments}

We would like to thank the staff at the Lung and Allergy research Unit, Skåne University hospital for assistance and Anders Olin for including patients in the Lund cohorts. We would like to thank Ida Åberg for excellent experimental help. The authors would like to thank the staff at the Respiratory Health Network Tissue Bank of the FRQS for their valuable assistance with the lung eQTL dataset at Laval University.

\section{Funding}

Ellen Tufvesson and The Lund cohort study was supported by independent research grants from the Swedish Heart and Lung Foundation, Crafoord Foundation, Evy and Gunnar Sandberg's Foundation and the Royal Physiographic Society in Lund. K. de Jong is supported by grant number 4.113.007 the Lung Foundation Netherlands. The LifeLines cohort study was supported by the Dutch Ministry of Health, Welfare and Sport, the Ministry of Economic Affairs, Agriculture and Innovation, the province of Groningen, the European Union (regional development fund), the Northern Netherlands
Provinces (SNN), the Netherlands Organization for Scientific Research (NWO), University Medical Center Groningen (UMCG), University of Groningen, de Nierstichting (the Dutch Kidney Foundation), and the Diabetes Fonds (the Diabetic Foundation). The Vlagtwedde-Vlaardingen cohort study was supported by the Ministry of Health and Environmental Hygiene of the Netherlands and the Netherlands Asthma Fund (grant 187) and the Netherlands Asthma Fund grant no. 3.2.02.51, the Stichting Astma Bestrijding, BBMRI-NL (Complementiation project), and the European Respiratory Society COPD research award 2011 to H.M. Boezen. Y. Bossé holds a Canada Research Chair in Genomics of Heart and Lung Diseases. A. Faiz holds a ERS RESPIRE2 fellowship.

\section{Availability of data and materials}

Please contact author for data request.

\section{Authors' contributions}

Concept and Design: JW, L, KJ, DP, AF, ET; Acquisition of data: JW, LJ, KJ, DS, YB, DN, JA, LB, AF, ET; Analysis and Interpretation: JW, LJ, KJ, JV, DP, AF, ET; Critical comments: All authors provided critical comments. All authors read and approved the final manuscript.

\section{Competing interests}

Dr. van den Berge reports research grants paid to University from GlaxoSmithkline, TEVA, and Chiesi, outside the submitted work; Dr. Sin reports grants and personal fees from AstraZeneca, personal fees from Boehringer Ingelheim, personal fees from Almirall, outside the submitted work; Dirkje S. Postma: The University of Groningen has received money for Professor Postma regarding a grant for research from Astra Zeneca, Chiesi, Genentec, GSK and Roche. Fees for consultancies were given to the University of Groningen by Astra Zeneca, Boehringer Ingelheim, Chiesi, GSK, Takeda and TEVA. None of the other authors have any conflict of interest.

\section{Consent for publication}

Not applicable

\section{Ethics approval and consent to participate}

All subjects signed written informed consent. The Regional Ethics Review Board in Lund approved the study on the Lund cohort (ref. 2008-431). The ethics committees of the Institut universitaire de cardiologie et de pneumologie de Québec and the UBC-Providence Health Care Research Institute Ethics Board approved the study for Laval and UBC, respectively. The study was approved by the Medical Ethics Committee of the University Medical Center Groningen, Groningen, The Netherlands (ref. METc 2007/152).

\section{Publisher's Note}

Springer Nature remains neutral with regard to jurisdictional claims in published maps and institutional affiliations.

\section{Author details}

${ }^{1}$ Respiratory Medicine and Allergology, Department of Clinical Sciences Lund, BMC, D12, Lund University, Skåne University Hospital, 22184 Lund, Sweden. ${ }^{2}$ University Medical Center Groningen, GRIAC (Groningen Research Institute for Asthma and COPD), Department of Epidemiology, University of Groningen, Groningen, The Netherlands. ${ }^{3}$ University Medical Center Groningen, Department of Pulmonology, GRIAC (Groningen Research Institute for Asthma and COPD), University of Groningen, Groningen, The Netherlands. ${ }^{4}$ Department of Medicine (Respirology), University of British Columbia, Centre for Heart Lung Innovation, Vancouver, Canada.

${ }^{5}$ Department of Molecular Medicine, Institut universitaire de cardiologie et de pneumologie de Québec, Laval University, Québec, Canada. ${ }^{6}$ Genetics and Pharmacogenomics (GpGx), Merck Research Laboratories, Boston, MA, USA.

Received: 16 February 2017 Accepted: 21 April 2017

Published online: 02 May 2017

\section{References}

1. (WHO) WHO. The top 10 causes of death [web page on the Internet. Geneva: WHO. http://www.who.int/mediacentre/factsheets/fs310/en/ [updated May 2014. Fact sheet number 310]. 
2. Kim WJ, Lee SD. Candidate genes for COPD: current evidence and research. Int J Chron Obstruct Pulmon Dis. 2015;10:2249-55.

3. Huang Q. Genetic study of complex diseases in the post-GWAS era. J Genet Genomics. 2015:42(3):87-98.

4. Lee JH, McDonald ML, Cho MH, Wan ES, Castaldi PJ, Hunninghake GM, et al. DNAH5 is associated with total lung capacity in chronic obstructive pulmonary disease. Respir Res. 2014;15:97.

5. Brandsma CA, van den Berge M, Postma DS, Jonker MR, Brouwer S, Pare PD, et al. A large lung gene expression study identifying fibulin-5 as a novel player in tissue repair in COPD. Thorax. 2015;70(1):21-32.

6. Hao K, Bosse Y, Nickle DC, Pare PD, Postma DS, Laviolette M, et al. Lung eQTLs to help reveal the molecular underpinnings of asthma. PLoS Genet. 2012;8(11):e1003029.

7. Dijkstra AE, Smolonska J, van den Berge M, Wijmenga C, Zanen P, Luinge MA, et al. Susceptibility to chronic mucus hypersecretion, a genome wide association study. PLoS One. 2014;9(4):e91621.

8. Chen X, Xu X, Xiao F. Heterogeneity of chronic obstructive pulmonary disease: from phenotype to genotype. Front Med. 2013;7(4):425-32.

9. Bossé Y. Updates on the COPD gene list. Int J Chron Obstruct Pulmon Dis. 2012;7:607-31.

10. Silverman EK, Sandhaus RA. Clinical practice. Alpha1-antitrypsin deficiency. N Engl J Med. 2009;360(26):2749-57.

11. Buono M, Cosma MP. Sulfatase activities towards the regulation of cell metabolism and signaling in mammals. Cell Mol Life Sci. 2010;67(5):769-80.

12. Cosma MP, Pepe S, Annunziata I, Newbold RF, Grompe M, Parenti G, et al. The multiple sulfatase deficiency gene encodes an essential and limiting factor for the activity of sulfatases. Cell. 2003;113(4):445-56.

13. Dierks T, Schmidt B, Borissenko LV, Peng J, Preusser A, Mariappan M, et al. Multiple sulfatase deficiency is caused by mutations in the gene encoding the human C(alpha)-formylglycine generating enzyme. Cell. 2003;113(4):435-44.

14. Cosma MP, Pepe S, Parenti G, Settembre C, Annunziata I, Wade-Martins R, et al. Molecular and functional analysis of SUMF1 mutations in multiple sulfatase deficiency. Hum Mutat. 2004;23(6):576-81.

15. Fraldi A, Biffi A, Lombardi A, Visigalli I, Pepe $S$, Settembre C, et al. SUMF1 enhances sulfatase activities in vivo in five sulfatase deficiencies. Biochem J. 2007;403(2):305-12.

16. Dickmanns A, Schmidt B, Rudolph MG, Mariappan M, Dierks T, von Figura K et al. Crystal structure of human pFGE, the paralog of the Calphaformylglycine-generating enzyme. J Biol Chem. 2005;280(15):15180-7.

17. Preusser-Kunze A, Mariappan M, Schmidt B, Gande SL, Mutenda K, Wenzel D, et al. Molecular characterization of the human Calphaformylglycine-generating enzyme. J Biol Chem. 2005;280(15):14900-10.

18. Diez-Roux G, Ballabio A. Sulfatases and human disease. Annu Rev Genomics Hum Genet. 2005:6:355-79.

19. Schlotawa L, Ennemann EC, Radhakrishnan K, Schmidt B, Chakrapani A, Christen HJ, et al. SUMF1 mutations affecting stability and activity of formylglycine generating enzyme predict clinical outcome in multiple sulfatase deficiency. Eur J Hum Genet. 2011;19(3):253-61.

20. Berger Kl, Fagondes SC, Giugliani R, Hardy KA, Lee KS, McArdle C, et al. Respiratory and sleep disorders in mucopolysaccharidosis. J Inherit Metab Dis. 2013;36(2):201-10.

21. Settembre C, Annunziata I, Spampanato C, Zarcone D, Cobellis G, Nusco E, et al. Systemic inflammation and neurodegeneration in a mouse model of multiple sulfatase deficiency. Proc Natl Acad Sci U S A. 2007;104(11):4506-11.

22. Arteaga-Solis E, Settembre C, Ballabio A, Karsenty G. Sulfatases are determinants of alveolar formation. Matrix Biol. 2012;31(4):253-60.

23. Macintyre N, Crapo RO, Viegi G, Johnson DC, van der Grinten CP, Brusasco V, et al. Standardisation of the single-breath determination of carbon monoxide uptake in the lung. Eur Respir J. 2005;26(4):720-35.

24. Miller MR, Hankinson J, Brusasco V, Burgos F, Casaburi R, Coates A, et al. Standardisation of spirometry. Eur Respir J. 2005;26(2):319-38.

25. Wanger J, Clausen JL, Coates A, Pedersen OF, Brusasco V, Burgos F, et al. Standardisation of the measurement of lung volumes. Eur Respir J. 2005;26(3):511-22

26. Crapo RO, Morris AH, Gardner RM. Reference spirometric values using techniques and equipment that meet ATS recommendations. Am Rev Respir Dis. 1981;123(6):659-64.

27. Quanjer PH, Tammeling GJ, Cotes JE, Pedersen OF, Peslin R, Yernault JC. Lung volumes and forced ventilatory flows. Report Working Party Standardization of Lung Function Tests. European Community for Steel and
Coal. Official Statement of the European Respiratory Society. Eur Respir J Suppl. 1993;16:5-40.

28. Tufvesson E, Aronsson D, Bjermer L. Cysteinyl-leukotriene levels in sputum differentiate asthma from rhinitis patients with or without bronchial hyperresponsiveness. Clin Exp Allergy. 2007;37(7):1067-73.

29. Tufvesson E, Nihlberg K, Westergren-Thorsson G, Bjermer L. Leukotriene receptors are differently expressed in fibroblast from peripheral versus central airways in asthmatics and healthy controls. Prostaglandins Leukot Essent Fat Acids. 2011:85(2):67-73.

30. Scholtens S, Smidt N, Swertz MA, Bakker SJ, Dotinga A, Vonk JM, et al. Cohort Profile: LifeLines, a three-generation cohort study and biobank. Int J Epidemiol. 2015;44(4):1172-80.

31. Jurinke $\mathrm{C}$, van den Boom D, Cantor CR, Koster H. Automated genotyping using the DNA MassArray technology. Methods Mol Biol. 2002;187:179-92.

\section{Submit your next manuscript to BioMed Central and we will help you at every step:}

- We accept pre-submission inquiries

- Our selector tool helps you to find the most relevant journal

- We provide round the clock customer support

- Convenient online submission

- Thorough peer review

- Inclusion in PubMed and all major indexing services

- Maximum visibility for your research

Submit your manuscript at www.biomedcentral.com/submit

) Biomed Central 